\title{
Research on a PMSM control system with microcontroller
}

\author{
Guozhe Yang ${ }^{\mathrm{a}}$, Feng Liu ${ }^{\mathrm{b}}$ \\ School of Mechanical Engineering, Shenyang University of Technology, Shenyang, PR China \\ 110870 \\ a yangguozhe@sina.comb zxsys12@126.com
}

Key words: PMSM; microcontroller; control

Abstract. This paper Application introduces a complete system level solution to drive a BLDC Motor for cordless power tools. To show the efficiency, present a thermal solution for a power stage with SMD MOSFETs and show how to implement the control with the microcontroller to drive a BLDC motor equipped with 3 hall sensors. This intend to reduce the system cost and improve the efficiency and increase the power density, which in turn provides longer run time and higher peak power pulse capability.

\section{Introduction}

The paper give a complete system solution implemented with a 3-phase bridge MOSFET power stage and a control system featuring Infineon's low cost 32-bit microcontroller XMC1302. This unit is specifically designed for cordless power tool application by using simple plug and play system, but it can easily be adopted for any 3-phase brushless DC motor application. Its compact design, optimized thermal performance and a ready to use firmware allows the user to effectively and quickly implement this design.

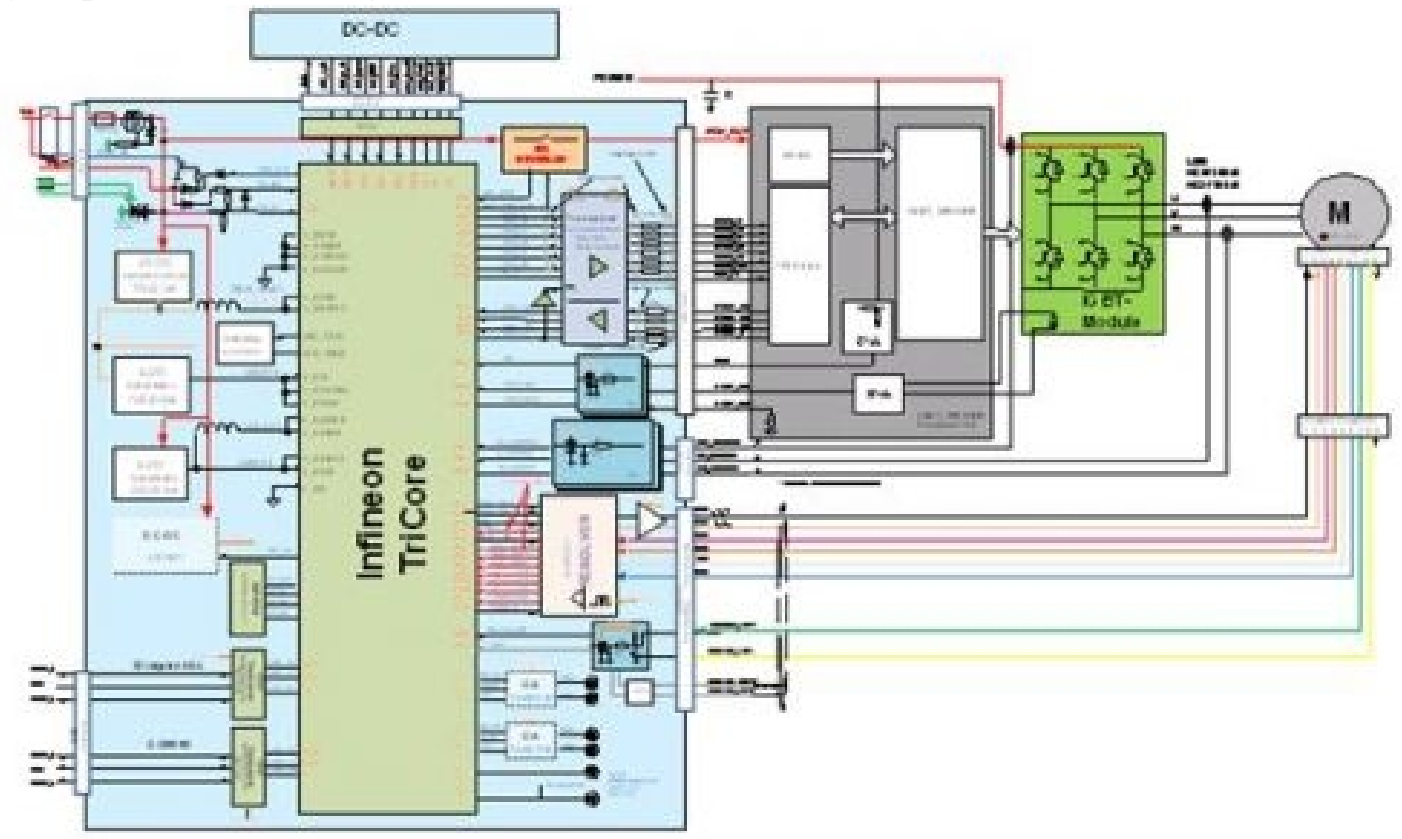

Fig 1 Overview of complete system

The software can be modified to implement additional features. The control system provides test points to all signals from the microcontroller. This allows the user to monitor the signals of interest. This system is more than just a reference design. Its unique layout, cooling concept and utilization 
of surface mount components will result in the highest power density, reliability, and a low cost product.

The XMC microcontroller is already programmed with dedicated software for power drill application. The following functions are implemented in the software that Block commutation for running BLDC motor with voltage control and Wrong Hall sensor shut down. The BLDC motor equipped with 3 hall sensors.

\section{Motor control solution}

The power stage is designed to drive a BLDC motor using two MOSFETs in parallel. The gate resistors have a value of $100 \mathrm{Ohm}$ which can be further modified depending on desired performance. Higher value of gate resistors will increase switching losses, since the MOSFETs are switching slower, but at the same time, the slower switching will generate lower voltage overshoots and require lower filter capacitors value. If the gate resistors values are reduced, it is possible to reduce the dead time between high and low side MOSFETs leading to faster switching and lower switching losses, but on the other hand, the parasitic inductance of the battery and the circuit parasitic inductance will have more effect and generate higher voltage overshoot. Multilayer ceramic caps which will filter out high frequency currents. The number of MLCCs can be further optimized depending on system requirements.

The current measurement is implemented by means of measuring the voltage across the Drain-Source of the MOSFET. Since the MOSFET behaves like a resistor when in "ON" state, the
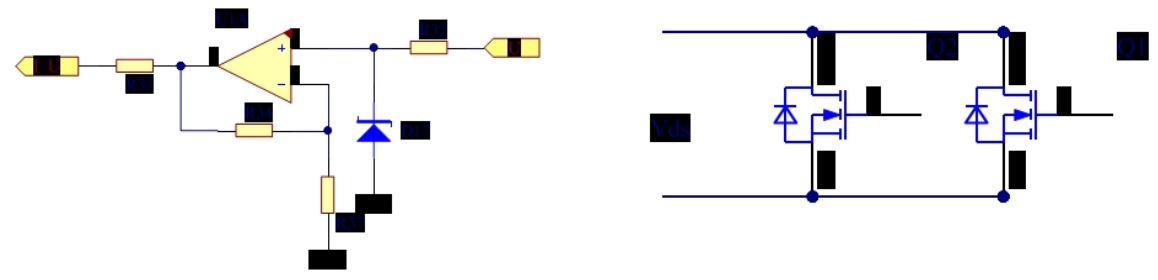

Fig 2 Current Measurements Schematics

voltage across it can be measured and the current through MOSFET can be calculated by using Ohm's law.

$$
I_{d s}=V_{d s} / R_{D s}(\text { on })
$$

Since the $\mathrm{R}_{\mathrm{DS}(\mathrm{on})}$ is dependent on temperature, we need to compensate for temperature change, we can see $R_{D S(o n)}$ dependency of temperature. This provided are for a typical and a maximum initial $R_{D S(o n)}$ value. The software which is included in this package, is assuming a linear $R_{D S(o n)}$ dependency of temperature. The temperature information is obtained from the temperature sensor. If more accurate current measurements are required, one can create a curve fit formula instead of linear approximation. Also in effort to reduce the measurement error, we can factor out the variation of initial $\mathrm{R}_{\mathrm{DS}(\mathrm{on})}$ and op-amp offset by initial calibration.

In order to compensate for temperature change we can calculate the $\mathrm{R}_{\mathrm{DS}(\mathrm{on}) \text {,hot }}$ as

$$
R_{D S(\text { on }), h o t}=R_{D S(\text { on }), \text { cold }} *\left(1+\alpha *\left(T_{\text {hot }}-T_{\text {cold }}\right)\right)
$$

where $\alpha$ is a temperature coefficient. The current is calculated as

$$
I_{\text {phase }}=V_{D S} / V_{D S(n), h o t}
$$


Since the measured $\mathrm{V}_{\mathrm{DS}}$ signal is small due to very low $\mathrm{R}_{\mathrm{DS}(\mathrm{on})}(0.6 \mathrm{mOhm})$, an op-amp is used to amplify this signal. The op-amp amplification is set to $30 \mathrm{~V} / \mathrm{V}$. The current information is used to detect overload condition and shut down the inverter.

The XMC1300 devices are members of the XMC1000 family of microcontrollers based on the $\mathrm{ARM}^{(\mathrm{R})}$ Cortex $^{(\mathrm{TM})}$-M0 processor core. The XMC1300 series is suitable for the real-time control needs of the BLDC motor. It also controls all input and output signals necessary for the power tool operation. XMC Microcontroller features has CPU Subsystem with Core of high performance 32-bit CPU and Most of 16-bit Thumb instruction set and Subset of 32-bit Thumb2 instruction set and High code density with 32-bit performance and Single cycle 32-bit hardware multiplier and System timer (SysTick) for Operating System support and Ultra low power consumption and Nested Vectored Interrupt Controller (NVIC) and Event Request Unit (ERU) for programmable processing of external and internal service requests and MATH Co-processor (MATH), consists of a CORDIC unit for trigonometric calculation and a division unit. Two Universal Serial Interface Channels (USIC), usable as UART, double-SPI, quad-SPI, IIC, IIS and LIN interfaces

Control System has safe system which has Window Watchdog Timer (WDT) for safety sensitive applications and Real Time Clock module with alarm support (RTC) and System Control Unit (SCU) for system configuration and control and Pseudo random number generator (PRNG), provides random data with fast generation times.

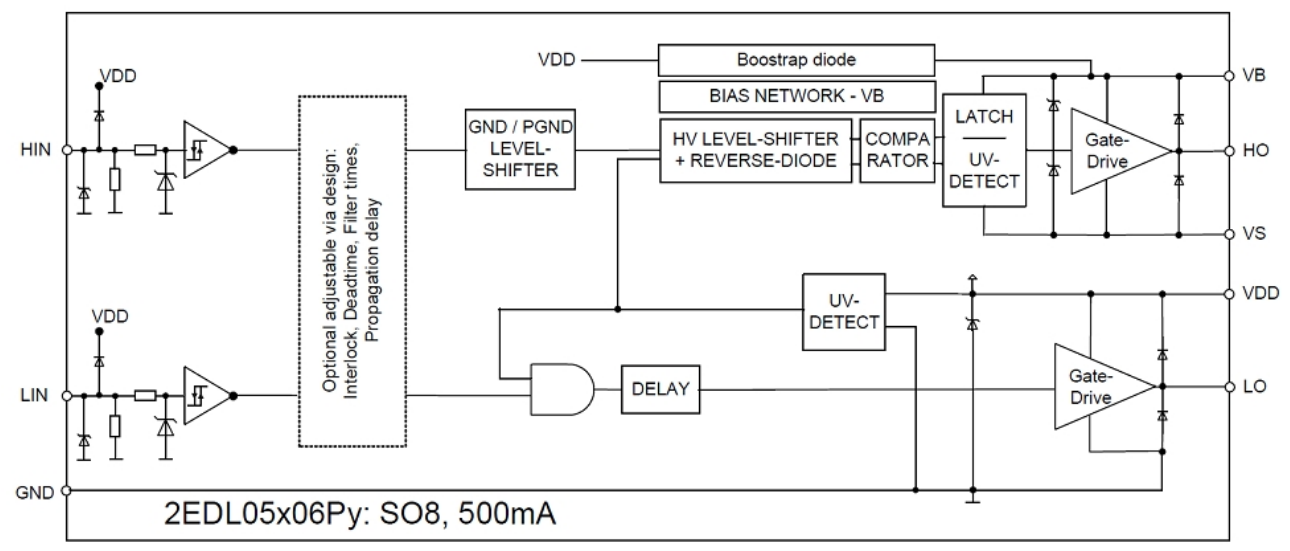

Fig 3 single gate driver block diagram

The motor control software is based on a voltage control block commutation algorithm of the BLDC motor. The microcontroller reads 3 hall switch patterns in order to determine the correct switching sequence for the phase commutation. Other input variables such as phase current, temperature, supply voltage, and a wrong hall pattern sequence are also being monitored by the microcontroller. According to their values, the microcontroller takes an action and controls the power inverter stage accordingly. This demonstration board is already programmed with dedicated software for cordless power drill and is ready for use.

\section{Efficiency measurement}

A regulated power supply is used to provide the voltage to the system. The supplied current and voltage is fed to the power analyzer. All 3 phase voltages and currents are fed to the power analyzer. The result of measurements are shown in fig 4 ..

In order to apply the load, another LOAD BLDC motor is used to break the BLDC MOTOR. These two motors are interconnected with a mechanical coupling. When the BLDC motor spins, the load motor acts as a generator and induces voltage which is rectified with a 3 phase diode bridge. This output voltage is further filtered with a DC link cap. A DC electronic load is connected at the 
output of the 3 phase diode bridge. By applying different setting on the electronic load, different loading conditions can be achieved.

The efficiency measurements and the temperature graphs show that the BSC010N04LSI has about $20 \%$ power loss reduction or $20 \%$ longer running time as compared to BSC010N04LS under the same loading conditions. This efficiency gain is due to integrated monolithic Schottky-like diode.

If we take a closer look at the diode forward voltage graph, we can see that the BSC010N04LSI diode has a lower forward voltage drop, and also lower recovery charge which account for higher efficiency.

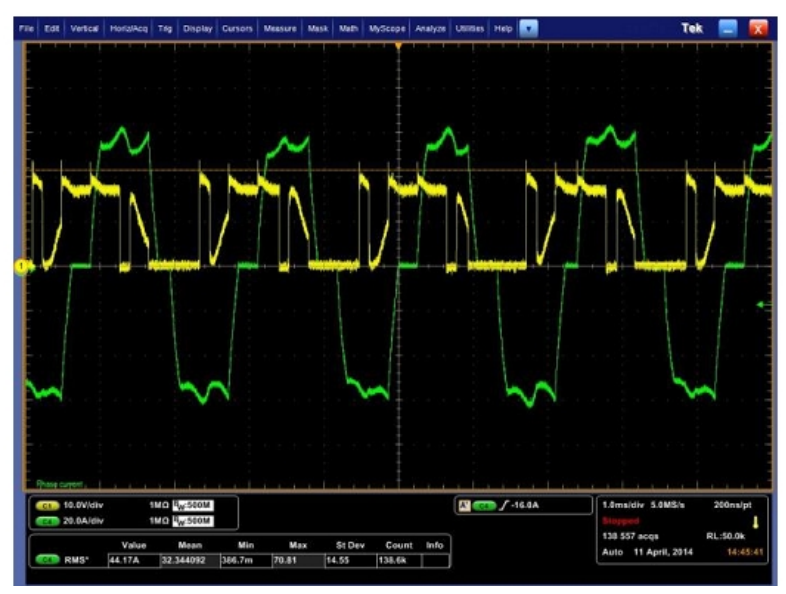

Fig 4 Phase voltage and phase current

\section{Conclusion}

The system provides a plug and play solution for driving BLDC motors in cordless power tool applications. The optimized size allows a quick integration in cordless power drills. With Infineon developed dedicated software, and a free software development platform DAVE(TM), the user is able to integrate additional functions. It was shown that in BLDC motor power inverter, the body diode plays a key role. The power loss and temperature measurements show an improvement of up to $20 \%$ of BSC010N04LSI MOSFET with integrated Schottky-like diode.

\section{References}

[1] G Z Yang, F Liu, H B Lin. Research on an Embedded Sevro Control system of micro-EDM. Applied Mechanics and Materials. vol 120, 2011, p.573-577

[2] Han Fuzhu, Wachi Shinya, Kunieda Masanori. Improvement of Machining Characteristics of Micro-EDM Using Transistor Type Isopulse Generator and Servo Feed Control. Precision Engineering, 2004, p.378-385.

[3] Matsuhara Y, Obara H. Study on High Finish Machining in Wire EDM. Journal of Electrical machining Technology, 2004, p.28:19-22.

[4] Naotake M, Hiromichi M, Nagao S. Development of an Electrical Discharge Drilling Device by Using a New Method for Direct Drive of Electrode. Journal of the Japan Society of Precision Engineering, 1992, p.2063-2068.

[5] Katsushi F, Naotake M, Toshiro H. Direct Drive Mechanism of EDM Electrode Utilizing Elliptical Movement. Journal of the Japan Society of Precision Engineering, 1995, p.672-676.

[6] Li Y, Guo M, Zhou Z Y, et al. Micro Electro Discharge Machine with an Inchworm Type of Micro Feed Mechanism. Journal of the International Societies for Precision Engineering and Nanotechnology, (2002), pp.7-14 